Article

\title{
The Association between Adult Weight Gain and Insulin Resistance at Middle Age: Mediation by Visceral Fat and Liver Fat
}

\author{
Inge Verkouter ${ }^{1}\left(0\right.$, Raymond Noordam ${ }^{2, *}$, Saskia le Cessie ${ }^{1,3}$, Rob M. van Dam ${ }^{4}$, \\ Hildo J. Lamb ${ }^{5}{ }^{\circledR}$, Frits R. Rosendaal ${ }^{1}$, Diana van Heemst ${ }^{2} \oplus$ and Renée de Mutsert ${ }^{1}$ \\ 1 Department of Clinical Epidemiology, Leiden University Medical Center, 2333 ZA Leiden, The Netherlands; \\ i.verkouter@lumc.nl (I.V.); S.le_Cessie@lumc.nl (S.L.C.); F.R.Rosendaal@lumc.nl (F.R.R.); \\ R.de_Mutsert@lumc.nl (R.D.M.) \\ 2 Department of Internal Medicine, Section Gerontology and Geriatrics, Leiden University Medical Center, \\ 2333 ZA Leiden, The Netherlands; D.van_Heemst@lumc.nl \\ 3 Department of Biomedical Data Sciences, Leiden University Medical Center, \\ 2333 ZA Leiden, The Netherlands \\ 4 Saw Swee Hock School of Public Health and Department of Medicine, Yong Loo Lin School of Medicine, \\ National University of Singapore and National University Health System, Singapore 117549, Singapore; \\ rob.van.dam@nus.edu.sg \\ 5 Department of Radiology, Leiden University Medical Center, 2333 ZA Leiden, The Netherlands; \\ H.J.Lamb@lumc.nl \\ * Correspondence: r.noordam@lumc.nl
}

Received: 14 August 2019; Accepted: 24 September 2019; Published: 28 September 2019

\begin{abstract}
We aimed to investigate the role of the amount of visceral fat and liver fat in the association between adult weight change and insulin resistance at middle age. In the Netherlands Epidemiology of Obesity study, adult weight change was calculated with recalled body weight at age 20 years and measured body weight at middle age. Measures of insulin resistance were calculated using both fasting and postprandial glucose and insulin concentrations. Visceral fat was assessed by magnetic resonance $(M R)$ imaging and liver fat by proton-MR spectroscopy $(N=1758)$. We examined the association between adult weight change and insulin resistance with linear regression, adjusted for confounding factors. To investigate mediation, we additionally adjusted for total body fat, visceral fat, and liver fat. In participants who gained $\geq 50 \%$ of body weight during adulthood, homeostatic model assessment for insulin resistance (HOMA-IR) was 3.22 (95\% CI 2.76; 3.77) times higher than in weight maintainers. In a joint model, total body fat mediated this association for $8.1 \%(95 \% \mathrm{CI}-9.2 ; 25.4)$, visceral fat for $32.0 \%(18.6 ; 45.4 \%)$ and liver fat for $22.5 \%(15.0 ; 30.1)$. The association between adult weight gain and insulin resistance at middle age is largely mediated by both visceral fat and liver fat.
\end{abstract}

Keywords: body weight changes; visceral fat; fatty liver; insulin resistance

\section{Introduction}

Adult weight gain and obesity are well-established causal risk factors for the development of type 2 diabetes mellitus, cardiovascular disease and obesity-related cancers [1-3]. In line with these findings, adult weight gain was strongly associated with increased insulin resistance in multiple studies [4-9].

It is well-established that abdominal adiposity, and in particular visceral adipose tissue, is strongly related to insulin resistance and risk of type 2 diabetes mellitus, also after adjustment for total body fat [10-15]. In a previous analysis, we observed that larger gain in body weight during adulthood was associated with more visceral fat and liver fat at middle age, compared with weight maintenance [16]. 
This finding is in agreement with the 'lipid overflow' hypothesis, which postulates that lipids are stored in the visceral area and in and around organs (ectopic fat) when the subcutaneous adipose tissue has reached its limited storage capacity $[17,18]$. In addition to visceral adipose tissue, excess liver fat has been associated with insulin resistance [19], as well as with a higher risk of type 2 diabetes mellitus, cardiovascular disease and mortality [20].

As both excess visceral fat and liver fat are strongly associated with increased insulin resistance and type 2 diabetes, we hypothesized that the association between adult weight change and insulin resistance is largely mediated by both excess visceral fat and liver fat. Therefore, the aim of this study was to investigate to what extent the association of adult weight change with insulin resistance is mediated by the amounts of visceral fat and liver fat at middle age.

\section{Methods}

\subsection{Study Design and Study Population}

The Netherlands Epidemiology of Obesity (NEO) study is a population-based cohort study of 6671 individuals aged 45-65 years, with an oversampling of individuals with body mass index (BMI) $\geq 27 \mathrm{~kg} / \mathrm{m}^{2}$, living in the greater area of Leiden (in the West of the Netherlands). All inhabitants aged between 45 and 65 years from one municipality (Leiderdorp) were invited to participate irrespective of their BMI, allowing for a reference distribution of BMI. The study design and population are described in detail elsewhere [21].

Participants were invited to a baseline visit at the NEO study center of the Leiden University Medical Center (LUMC) after an overnight fast. Prior to the study visit, participants completed a general questionnaire at home to report demographic, lifestyle and clinical information. At the study center, participants completed a screening form, asking about anything that might create a health risk or interfere with magnetic resonance imaging, e.g., presence of metallic devices, claustrophobia and a body circumference $>1.70 \mathrm{~m}$. Of the eligible participants, 2580 participants were randomly selected to undergo magnetic resonance imaging(MRI) [21]. This subset of participants from the NEO cohort has similar characteristics as the participants from the NEO cohort not participating in this subset, apart from a slightly higher BMI and more individuals with a history of cardiovascular disease in the subset that did not participate [15]. The Medical Ethical Committee of the LUMC approved the NEO study. All participants provided written informed consent.

For the present study, we performed cross-sectional analyses with baseline measurements of the NEO study. Hepatic triglyceride content was available in 2086 of the participants who underwent MRI, due to technical failures or an insufficient quality of the measurements to estimate liver fat content. We excluded participants with images of insufficient quality to estimate visceral adipose tissue $(n=11)$, with missing data on recalled body weight at age 20 years $(n=60)$, and with a BMI at age 20 below $14.0 \mathrm{~kg} / \mathrm{m}^{2}(n=1)$. Additionally, we excluded participants who used glucose-lowering medication $(n=129)$, were non-fasting at baseline $(n=1)$, or had missing data on postprandial glucose at $30 \mathrm{~min}$ $(n=38)$, at $150 \mathrm{~min}(n=30)$ or fasting insulin $(n=3)$, total body fat at baseline $(n=3)$, ethnicity $(n=2)$, educational level $(n=15)$ and physical activity $(n=35)$, resulting in 1758 participants $(913$ men, BMI range $20.1-39.6 \mathrm{~kg} / \mathrm{m}^{2}$ and 845 women, BMI range $18.2-45.3 \mathrm{~kg} / \mathrm{m}^{2}$ ) who were included in the analyses. The majority of our study population was of Caucasian ethnicity $(96.3 \%)$. Other ethnicities that were represented in our study sample are African (0.5\%), Turkish (0.3\%), South-East Asian (0.5\%), Hindu $(0.1 \%)$, and ethnicities other than aforementioned $(2.3 \%)$.

\subsection{Data Collection}

\subsubsection{Weight Change during Adulthood}

At the baseline study visit, height without shoes was measured with a vertically fixed, calibrated tape measure. Body weight was measured and percent body fat was estimated by the Tanita bio 
impedance balance (TBF-310, Tanita International Division, UK) without shoes and $1 \mathrm{~kg}$ was subtracted to correct for weight of clothing. BMI at baseline was calculated by dividing the weight in $\mathrm{kg}$ by the height in meters squared.

Recalled weight at the age of 20 years was based on self-report. The general questionnaire included the question 'How much did you weigh (approximately) when you were 20 years old?'. BMI at age 20 years was calculated by dividing body weight at age $20 \mathrm{in} \mathrm{kg}$ by the height in meters squared at middle age with the assumption that height did not majorly change during adulthood. Relative weight change was calculated by subtracting weight at age 20 from measured baseline weight, divided by weight at age 20 , and multiplied by $100 \%$ [16].

\subsubsection{Visceral Fat and Liver Fat at Middle Age}

Visceral adipose tissue was directly assessed by MRI (1.5 Tesla MR imaging, Philips Medical Systems, Best, Netherlands) using a turbo spin echo imaging protocol with the following imaging parameters: 300/20; flip angle, $90^{\circ}$; section thickness, $10 \mathrm{~mm}$, section gap, $2 \mathrm{~mm}$. At the level of the fifth lumbar vertebra, three transverse slices were obtained during one breath-hold [21]. Imaging parameters were: $\mathrm{TR}=300 \mathrm{~ms} ; \mathrm{TE}=20 \mathrm{~ms}$; flip angle $=90^{\circ}$; slice thickness $=10 \mathrm{~mm}$, slice gap $=2 \mathrm{~mm}$. Visceral fat areas were quantified by converting the number of pixels to centimeters squared for all three slices and totaling the areas of the three sections, using in-house-developed software (MASS; Leiden University Medical Center, Leiden, the Netherlands).

Hepatic triglyceride content was quantified using ${ }^{1} \mathrm{H}$-magnetic resonance spectroscopy of the liver [21]. An $8 \mathrm{~mL}$ voxel was positioned in the right lobe of the liver, avoiding gross vascular structures and adipose tissue depots. Sixty-four averages were collected with water suppression (repetition time $=2900 \mathrm{~ms}$; echo time $=23 \mathrm{~ms}$ (2900/23). Data points (1024) were collected by using a 1000-Hz spectral line. Without changing any parameters, spectra without water suppression, with a repetition time of $10 \mathrm{~s}$ and with four averages were obtained as internal reference. Hepatic triglyceride content relative to water was calculated as (signal amplitude of triglyceride)/(signal amplitude of water) $\times 100$. Spectra were not corrected for frequency drift. Spectral data were analyzed while blinded to all study parameters, including age, sex, LV function and dimensions, BMI, waist circumference, visceral adipose tissue, and total body fat. Spectra were initially included when automatic fitting was successful. When line shapes were distorted by eddy currents or as a result of poor shimming, spectral data were rejected.

\subsubsection{Measures of Insulin Resistance at Middle Age}

Fasting blood samples were drawn from the antecubal vein after $5 \mathrm{~min}$ rest of the participant, after an overnight fast of at least $10 \mathrm{~h}$. Within $5 \mathrm{~min}$ after drawing a fasting blood sample, all participants consumed a liquid mixed meal $(400 \mathrm{~mL})$ that contained 2.5 megajoule (MJ), of which 16 percent of energy (En\%) was derived from protein, $50 \mathrm{En} \%$ from carbohydrates and $34 \mathrm{En} \%$ from fat. Subsequently, blood samples were drawn after 30 and $150 \mathrm{~min}$. Plasma glucose concentrations were determined by enzymatic and colorimetric methods (Roche Modular Analytics P800, Roche Diagnostics, Mannheim, Germany; $\mathrm{CV}<5 \%$ ) and serum insulin concentrations were determined by an immunometric method (Siemens Immulite 2500, Siemens Healthcare Diagnostics, Breda, The Netherlands; CV $<5 \%$ ) at the Department of Clinical Chemistry and Laboratory Medicine of the LUMC [21].

From fasting glucose and insulin concentrations, we calculated the Homeostasis Model Assessment for Insulin Resistance (HOMA-IR), a marker of hepatic insulin resistance [22]. HOMA-IR was calculated as fasting insulin $(\mu \mathrm{U} / \mathrm{mL}) \times$ fasting glucose $(\mathrm{mmol} / \mathrm{L}) / 22.5$ [22,23]. Matsuda Insulin Sensitivity Index (ISI) was calculated as 10,000/square root (fasting glucose $(\mathrm{mg} / \mathrm{dL}) \times$ fasting insulin $(\mu \mathrm{U} / \mathrm{mL})) \times$ $\left(\right.$ mean $_{\text {glucose0-150 }} \times$ mean $_{\text {insulin0-150 }}$ [24-26].

\subsubsection{Covariates}

Ethnicity was self-identified in the questionnaire and regrouped into Caucasian and other. Highest level of education was reported in ten categories according to the Dutch education system and regrouped 
in two categories: Low education (no education, primary school or lower vocational education) and high education (higher vocational education, university and postgraduate education). Smoking status was self-reported. Alcohol consumption was reported on the food frequency questionnaire and expressed as grams/day [27]. Participants reported the frequency and duration of their usual physical activity during leisure time in the Short questionnaire to assess health-enhancing physical activity (SQUASH), which was expressed in hours per week of metabolic equivalents [28,29]. Family history of diabetes mellitus and myocardial infarction were reported as having any parent or sibling with diabetes mellitus or not (reference group).

\subsection{Statistical Analyses}

In the NEO study, individuals with BMI $\geq 27 \mathrm{~kg} / \mathrm{m}^{2}$ are oversampled. To correctly represent associations for the general population [30], adjustments for the oversampling were made. This was done by weighting all participants towards the BMI distribution of participants from the Leiderdorp municipality [31], whose BMI distribution was similar to the BMI distribution of the general Dutch population [32]. All results were based on weighted analyses and are therefore generalizable to a population-based study without oversampling of individuals with $B M I \geq 27 \mathrm{~kg} / \mathrm{m}^{2}$ [21]. As a consequence of the weighting procedure, numbers of participants per category are presented as percentages.

Characteristics of the study population at middle age were expressed as mean (SD), median (25th, 27th percentiles or range) or as percentage, stratified by categories of weight change. We explored the distribution of weight change in the reference population of Leiderdorp in an earlier study, and observed that the majority of the population gained weight between age 20 years and middle age in this era [16]. Therefore, we decided to stratify categories of adult weight change as: weight loss of more than $5 \%$, weight change between $-5 \%$ and $5 \%$ (weight maintenance: reference category), weight gain of $5 \%$ to $25 \%, 25 \%$ to $50 \%$, and $\geq 50 \%$.

We performed linear regression analyses to examine the associations of adult weight change with fasting and postprandial glucose and insulin concentrations and with measures of insulin resistance at middle age, compared with the reference category of weight maintenance during adulthood. Potential confounding factors were defined a priori based on biological knowledge from previous studies. Crude models were adjusted for sex and age (model 1 ). In model 2, we additionally adjusted for BMI at age 20, because the percentage of weight change since age 20 depends on initial BMI at age 20. In model 3 , we additionally adjusted for ethnicity, education, smoking, alcohol consumption, physical activity, and family history of diabetes. Because of a skewed distribution, values of fasting and postprandial glucose and insulin, HOMA-IR and Matsuda ISI were all transformed to the natural logarithm. Regression coefficients and corresponding $95 \%$ confidence intervals (95\% CI) were back transformed and expressed as ratios, which can be interpreted as the relative changes in insulin resistance in a certain weight change category, compared with insulin resistance in the reference category of weight maintenance. For example: a ratio of 2 for HOMA-IR in individuals who gained $5 \%$ to $25 \%$ of body weight during adulthood indicates that these individuals have twofold higher HOMA-IR values at middle age than individuals who maintained their body weight during adulthood.

Subsequently, we examined mediation in the association between adult weight change and insulin resistance at middle age by total body fat, visceral fat, and liver fat at middle age according to the method proposed by Baron and Kenny [33]. This method is based on comparing the regression coefficient of the association between an exposure and outcome, and the regression coefficient of the association between the exposure and outcome adjusted for the mediating variable. First, we checked whether the exposure-outcome, exposure-mediator, and mediator-outcome associations were present in our study. Associations between adult weight change and total body fat, visceral fat, and liver fat were previously described for the NEO population [16], as well as the associations between total body fat, visceral fat, and liver fat and insulin resistance (HOMA-IR) [15]. The association between adult weight change and insulin resistance was examined in the present study. Secondly, we checked the 
assumption of no interaction between the exposure and mediator by examining the interaction between adult weight change and total body fat, visceral adipose tissue and hepatic triglyceride content in the association with insulin resistance. We attempted to avoid mediator-outcome confounding in the mediation analyses by adjusting for measured potential confounding factors.

Additional to Baron and Kenny's mediation method, we used structural equation modelling (SEM) to evaluate the effect of several mediators on the relation between adult weight change and insulin resistance adjusted for possible confounding factors, expressed as a percentage of mediation [34]. By multiplying the regression coefficients of the exposure-mediator and the mediator-outcome model, we were able to calculate the separate and combined indirect effects of total body fat, visceral fat and liver fat on the association between adult weight change and insulin resistance, with their corresponding $95 \%$ CIs. We divided the indirect effects by the total effect, which is the sum of the direct and indirect effects, to calculate the percentage of mediation by the different mediators and corresponding $95 \% \mathrm{CI}$.

We also conducted several sensitivity analyses. We repeated all analyses with absolute adult weight change between age 20 years and middle age in kilograms, instead of the relative weight change as a percentage. We repeated the analyses with relative weight change as a continuous variable. Additionally, we assessed whether there was interaction by sex in the association between adult weight change and insulin resistance by including a product term of sex and adult weight change, and repeated all analyses for men and women separately.

\section{Results}

\subsection{Characteristics of the Study Population}

A total of 1758 individuals ( $54 \%$ women) were analyzed in the present study. Mean (SD) age of the study population was 55 (6) years, mean BMI at age 20 years was $21.8(2.6) \mathrm{kg} / \mathrm{m}^{2}$, mean BMI at middle age was $25.8(3.9) \mathrm{kg} / \mathrm{m}^{2}$, and mean percentage of adult weight change was a gain in weight of $19.1 \%(16.0 \%)$.

Characteristics of the study population stratified by five weight change categories are presented in Table 1 . The proportion of women was higher in participants who gained more than $25 \%$ of body weight between age 20 and middle age than in participants who gained less than $25 \%$ during adulthood. Additionally, participants who gained more than $25 \%$ of body weight were less likely to be highly educated, and waist circumference, total body fat, visceral fat and liver fat at middle age were higher than in participants who gained less than $25 \%$ of body weight, in both men and women. Median fasting plasma glucose, fasting serum insulin and HOMA-IR were higher in individuals who gained more than $25 \%$ of body weight, compared with individuals who gained less than $25 \%$ of body weight, whereas Matsuda ISI was lower. 
Table 1. Characteristics of participants of the Netherlands Epidemiology of Obesity (NEO) study, aged 45 to 65 years, with measurements of visceral adipose tissue and hepatic triglyceride content by magnetic resonance imaging and spectroscopy, stratified by adult weight change $(N=1758)$.

\begin{tabular}{|c|c|c|c|c|c|}
\hline & Loss of $>5 \%$ & $\begin{array}{l}\text { Weight Maintenance } \\
-5 \% \text { to }<5 \%\end{array}$ & Gain of $\geq 5 \%$ to $<25 \%$ & Gain of $\geq 25 \%$ to $<50 \%$ & Gain of $\geq \mathbf{5 0} \%$ \\
\hline Proportion of population (\%) & 4.5 & 11.2 & 55.0 & 24.7 & 4.6 \\
\hline Sex (\% men) & 22 & 39 & 50 & 46 & 38 \\
\hline \multicolumn{6}{|l|}{ Body weight at age 20} \\
\hline Recalled weight at age $20(\mathrm{~kg})$ & $73.7(8.0)$ & $67.2(7.6)$ & $66.2(9.3)$ & $64.5(14.1)$ & $59.0(14.5)$ \\
\hline BMI at age $20\left(\mathrm{~kg} / \mathrm{m}^{2}\right)$ & $25.1(2.1)$ & $22.6(1.9)$ & $21.7(1.9)$ & $21.3(3.3)$ & $19.9(4.1)$ \\
\hline Change in weight (\%, range) & $-7.6(-32.2 ;-5.8)$ & $1.9(-4.9 ; 4.8)$ & $14.9(5.0 ; 24.9)$ & $32.3(25.0 ; 49.8)$ & $57.2(50.0 ; 102.8)$ \\
\hline \multicolumn{6}{|l|}{ Characteristics at middle age } \\
\hline Age (years) & $53(3)$ & $57(4)$ & $55(5)$ & $55(7)$ & $56(8)$ \\
\hline Ethnicity (\% Caucasian) & 100 & 96 & 96 & 98 & 91 \\
\hline Education (\% high) & 45 & 53 & 51 & 37 & 31 \\
\hline Smoking (\% current) & 19 & 21 & 13 & 13 & 11 \\
\hline Alcohol (g/day) & $4(1-21)$ & $10(4-16)$ & $11(3-23)$ & $8(2-21)$ & $8(1-21)$ \\
\hline Physical activity (MET-hours/week) & $27(19-58)$ & $42(28-56)$ & $31(17-53)$ & $26(14-44)$ & $20(10-42)$ \\
\hline Body weight $(\mathrm{kg})$ & $66.0(6.2)$ & $67.9(7.8)$ & $76.0(11.2)$ & $86.3(18.7)$ & $94.6(23.3)$ \\
\hline BMI $\left(\mathrm{kg} / \mathrm{m}^{2}\right)$ & $22.4(1.4)$ & $22.8(1.9)$ & $25.0(2.4)$ & $28.5(4.6)$ & $31.9(6.6)$ \\
\hline Waist circumference $(\mathrm{cm}, \mathrm{M} / \mathrm{W})$ & $90(5) / 76(5)$ & $87(6) / 77(7)$ & $96(7) / 82(7)$ & 105(12)/93(13) & $110(14) / 103(16)$ \\
\hline Total body fat $(\%, \mathrm{M} / \mathrm{W})$ & $18(3) / 32(3)$ & $20(3) / 31(4)$ & $24(3) / 35(4)$ & $28(7) / 41(6)$ & $31(8) / 44(10)$ \\
\hline Visceral adipose tissue $\left(\mathrm{cm}^{2}, \mathrm{M} / \mathrm{W}\right)$ & $50(44-66) / 21(14-37)$ & $50(19-79) / 36(24-47)$ & $98(76-133) / 49(35-69)$ & $135(104-173) / 88(59-113)$ & $158(131-210) / 118(94-156)$ \\
\hline $\begin{array}{l}\text { Hepatic triglyceride content }(\%, \mathrm{M} / \mathrm{W}) \\
\text { In women a. }\end{array}$ & 2.2(0.9-2.6)/0.9(0.7-1.6) & $1.8(1.0-3.6) / 1.2(0.7-1.7)$ & $3.5(2.0-7.0) / 1.6(1.1-3.6)$ & $6.0(3.5-14.0) / 3.4(1.6-8.4)$ & $11.8(3.8-20.8) / 7.7(3.7-18.8)$ \\
\hline Postmenopausal (\% yes) & 37 & 78 & 51 & 66 & 69 \\
\hline $\begin{array}{l}\text { Current use of sex hormones }{ }^{\mathrm{b}}(\%) \\
\text { Insulin resistance at middle age }\end{array}$ & 4 & 3 & 11 & 7 & 3 \\
\hline Family history of diabetes (\% yes) & 31 & 22 & 26 & 24 & 31 \\
\hline Family history of myocardial infarction (\% yes) & 26 & 34 & 39 & 47 & 48 \\
\hline Fasted plasma glucose $(\mathrm{mmol} / \mathrm{L})$ & $4.8(4.5-5.1)$ & $5.1(4.8-5.3)$ & $5.2(4.9-5.6)$ & $5.5(5.2-5.9)$ & $5.6(5.3-6.1)$ \\
\hline Fasted serum insulin (mU/L) & $5.5(4.1-6.5)$ & $5.4(3.6-7.1)$ & $7.3(5.2-9.9)$ & $10.5(7.6-14.7)$ & $13.0(8.6-21.6)$ \\
\hline HOMA-IR & $1.1(0.8-1.5)$ & $1.2(0.8-1.6)$ & $1.7(1.2-2.4)$ & $2.6(1.8-3.7)$ & $3.2(2.1-5.5)$ \\
\hline Matsuda ISI & $2.4(2.1-2.7)$ & $2.1(1.8-2.5)$ & $1.8(1.5-2.1)$ & $1.4(1.0-1.8)$ & $1.1(0.6-1.6)$ \\
\hline
\end{tabular}

$54 \%$ of study population. ${ }^{\mathrm{b}}$ Use of sex hormones included oral contraceptive and hormonal replacement therapy. Results were based on analyses weighted towards the BMI distribution resistance; Matsuda ISI, Matsuda insulin sensitivity index. Data are presented as mean (SD), median (25th-75th percentile/range) or percentage. 


\subsection{Adult Weight Change and Insulin Resistance at Middle Age}

As shown in Figure 1, each higher category of change in body weight during adulthood was associated with higher fasting and postprandial glucose and insulin concentrations at middle age, after adjustment for sex, age, BMI at age 20, ethnicity, education, smoking, alcohol consumption, physical activity, and family history of diabetes.
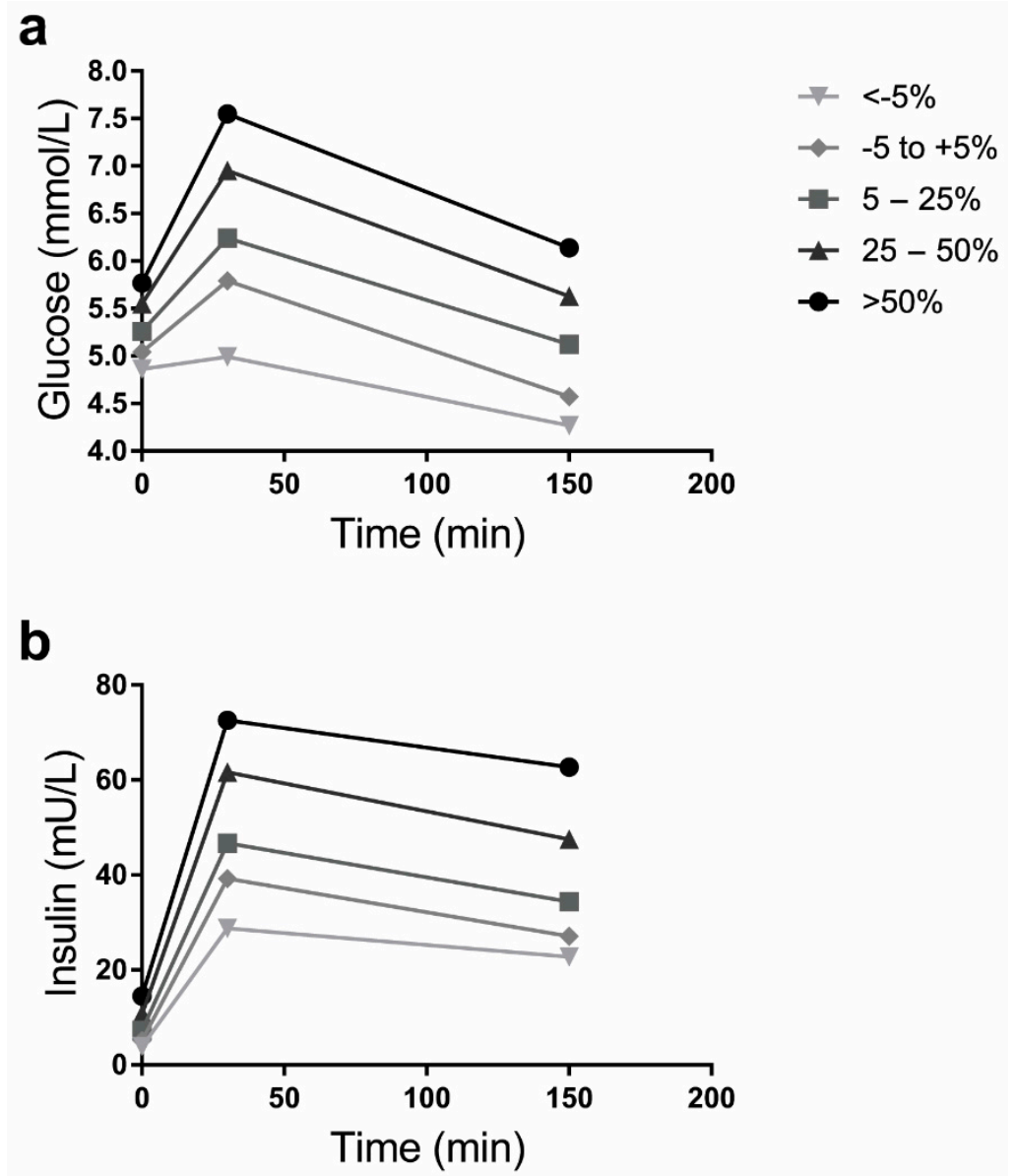

Figure 1. Estimated means of (a) glucose $(\mathrm{mmol} / \mathrm{L})$ and $(\mathbf{b})$ insulin $(\mathrm{mU} / \mathrm{L})$ blood concentrations fasting, and at $t=30$ and $t=150 \mathrm{~min}$ after a mixed meal challenge, stratified by adult weight change $(N=1758)$ and adjusted for sex, age, BMI at age 20, ethnicity, education, smoking, alcohol consumption, physical activity, and family history of diabetes.

After adjustment for potential confounding factors (model 2, Table 2), HOMA-IR was 1.37 (95\% CI $1.20 ; 1.56)$ times higher in participants who gained $5-25 \%$ of body weight, $2.04(1.79 ; 2.34)$ times higher in participants who gained $25-50 \%$ of body weight, and $2.65(2.24 ; 3.14)$ times higher in those who gained $\geq 50 \%$ of body weight during adulthood than in weight maintainers. The ratio of relative change in the Matsuda ISI was 0.76 (95\% CI 0.68 ; 0.84) in participants who gained $5-25 \%$ of body weight during adulthood, $0.51(0.46 ; 0.58)$ in participants who gained $25-50 \%$ of body weight compared with weight maintainers, and $0.40(0.34 ; 0.47)$ in participants who gained $\geq 50 \%$ of body weight in model 2 . After additional adjustment for BMI at age 20 (model 3), associations between adult weight change and both HOMA-IR and Matsuda ISI became slightly stronger (Table 2). Adjustment for sex, age and BMI at age 20 had the largest impact on the associations between adult weight gain and insulin resistance. 
Table 2. Relative change with $95 \%$ confidence intervals in measures of insulin resistance and insulin sensitivity for categories of weight change during adulthood, compared with weight maintenance $(N=1758)$.

\begin{tabular}{ccccccc}
\hline & \multicolumn{2}{c}{ Model 1 } & \multicolumn{2}{c}{ Model 2 } & \multicolumn{2}{c}{ Model 3 } \\
\cline { 2 - 7 } & Ratio & $\mathbf{9 5 \%}$ CI & Ratio & $\mathbf{9 5 \%}$ CI & Ratio & $\mathbf{9 5 \% ~ C I ~}$ \\
\hline HOMA-IR & & & & & & \\
$<-5.0 \%$ & 0.85 & $0.56 ; 1.30$ & 0.85 & $0.56 ; 1.30$ & 0.73 & $0.47 ; 1.12$ \\
$-5 \%$ to 5\% (ref) & 1 & & 1 & & 1 & \\
5-25\% & 1.38 & $1.21 ; 1.57$ & 1.37 & $1.20 ; 1.56$ & 1.47 & $1.30 ; 1.67$ \\
25-50\% & 2.14 & $1.87 ; 2.44$ & 2.04 & $1.79 ; 2.34$ & 2.28 & $2.01 ; 2.59$ \\
>50\% & 2.78 & $2.34 ; 3.30$ & 2.65 & $2.24 ; 3.14$ & 3.22 & $2.76 ; 3.77$ \\
Matsuda ISI & & & & & & \\
$<-5.0 \%$ & 1.22 & $0.92 ; 1.63$ & 1.23 & $0.92 ; 1.63$ & 1.40 & $1.05 ; 1.86$ \\
-5 to 5\% (ref) & 1 & & 1 & & 1 & \\
$5-25 \%$ & 0.75 & $0.67 ; 0.84$ & 0.76 & $0.68 ; 0.84$ & 0.71 & $0.64 ; 0.79$ \\
25-50\% & 0.49 & $0.44 ; 0.55$ & 0.51 & $0.46 ; 0.58$ & 0.47 & $0.42 ; 0.52$ \\
$>50 \%$ & 0.38 & $0.32 ; 0.44$ & 0.40 & $0.34 ; 0.47$ & 0.34 & $0.30 ; 0.39$ \\
\hline
\end{tabular}

Results were based on analyses weighted towards the BMI distribution of the general population and were derived from beta coefficients with $95 \%$ confidence intervals from linear regression analyses and expressed as ratios of outcome measures compared with weight maintenance during adulthood. Abbreviations: CI, confidence interval; HOMA-IR, homeostatic model assessment insulin resistance; Matsuda ISI, Matsuda insulin sensitivity index; ref, reference group. Model 1: Adjusted for sex and age; 2: additionally adjusted for ethnicity, education, smoking, alcohol consumption, physical activity and family history of diabetes; 3: additionally adjusted for BMI at age 20.

When using absolute adult weight change (in $\mathrm{kg}$ ) instead of relative weight change, results were similar (Table S1). Additionally, we repeated the analyses with adult weight change as continuous variable (per 10\% of weight change, Table S2), showing similar results.

\subsection{Mediation Analyses}

We did not observe interaction between the mediators (total body fat, visceral adipose tissue and hepatic triglyceride content) and adult weight change in the association with HOMA-IR or Matsuda ISI (Table S3).

As presented in Figure 2 and Table S4, after adjustment for total body fat at middle age, the association between adult weight change and HOMA-IR attenuated across all weight change categories. For example, individuals who gained $\geq 50 \%$ of body weight during adulthood had 1.87 $(1.48 ; 2.36)$ times higher mean HOMA-IR values. After additional adjustment for visceral fat, the association attenuated further to $1.49(1.17 ; 1.89)$, while after adjustment for liver fat to $1.59(1.27 ; 2.00)$. When we adjusted the association between adult weight change and HOMA-IR for total body fat, visceral fat and liver fat in one model, the association was $1.38(1.09 ; 1.74)$ for individuals who gained $\geq 50 \%$ of body weight. For Matsuda ISI, the pattern of associations was in opposite direction, but similar to HOMA-IR (Figure 2 and Table S4). 
a

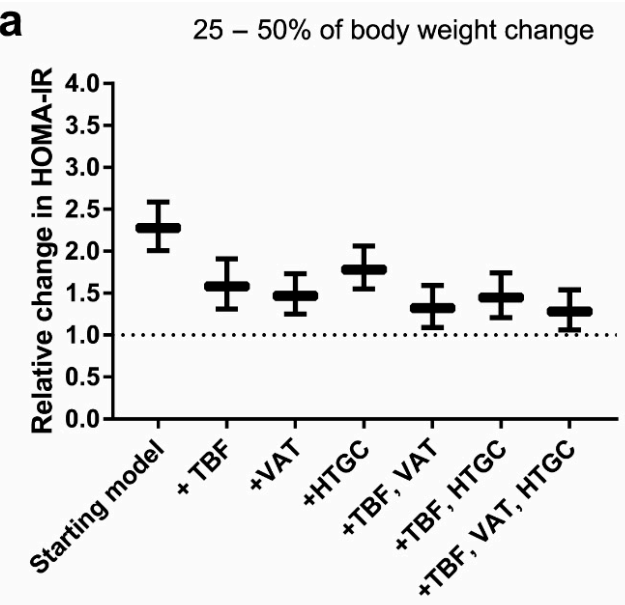

C

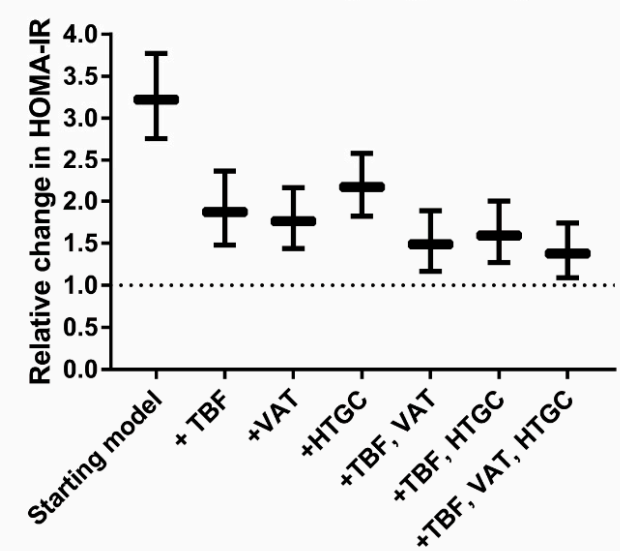

b

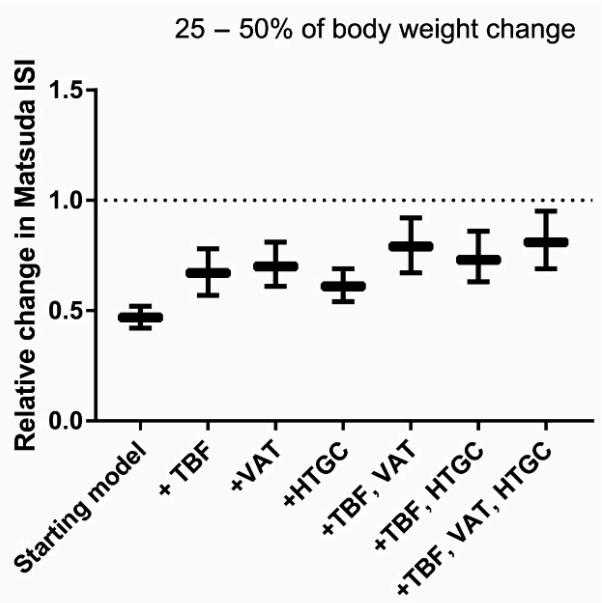

d

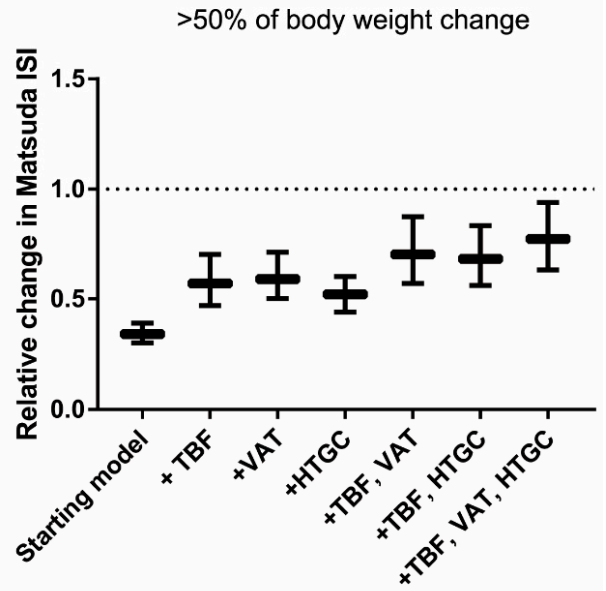

Figure 2. Relative changes ( $95 \% \mathrm{CI}$ ) of measures of HOMA-IR and Matsuda Index for participants who gained $25-50 \%$ of body weight $(\mathbf{a}, \mathbf{b})$ or $>50 \%$ of body weight $(\mathbf{c}, \mathbf{d})$ after addition of mediators, compared to weight maintainers. TBF, total body fat; VAT, visceral adipose tissue; HTGC, hepatic triglyceride content. Starting model (model 3) was adjusted for sex, age, BMI at age 20, ethnicity, education, smoking, alcohol consumption, physical activity and family history of diabetes.

Results for sex-stratified mediation analyses are presented in Table S5 (men) and Table S6 (women). We observed similar patterns of mediation by visceral fat and liver fat in men and women. Additionally, we did not observe interaction by sex in the association between adult weight change and HOMA-IR or Matsuda ISI ( $p$-value for interaction 0.53 and 0.67 , respectively).

In addition, we also performed SEM analyses to estimate the indirect effects of adult weight change through total body fat, visceral fat and liver fat as a percentage of its total effect (Table 3). Separately, total body fat, visceral adipose tissue and hepatic triglyceride content all had an indirect effect in the association between adult weight change and HOMA-IR (Table 3). However, when the joint mediating effect of total body fat and visceral fat or liver fat was considered, the indirect effect via total body fat disappeared in women, but remained in men. When all three mediators were included in the model, the percentage of mediation of the total association between adult weight change and HOMA-IR was $32.0 \%(95 \%$ CI $18.6 ; 45.4)$ for visceral fat and $22.5 \%(15.0 ; 30.1)$ for liver fat. Similar percentages of mediation were observed for Matsuda ISI (results not shown). 
Table 3. Analysis of indirect effects of the mediators total body fat, visceral adipose tissue and hepatic triglyceride content in the association between adult weight gain and HOMA-IR.

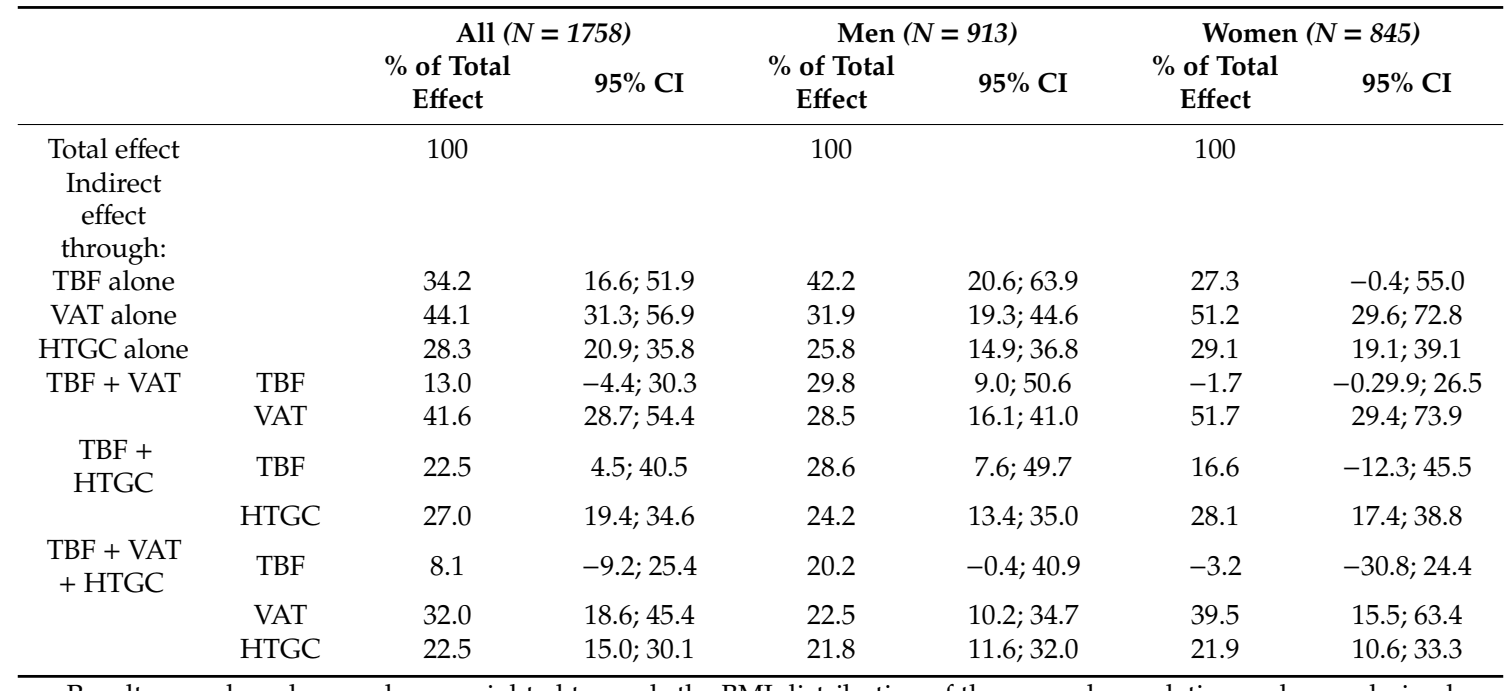

Results were based on analyses weighted towards the BMI distribution of the general population and were derived from multiplied path coefficients with $95 \%$ confidence intervals from structural equation modelling (path analysis) and expressed as indirect effects in the association between adult weight change (per 10\% weight change) and insulin resistance. Indirect effects were divided by total effects to calculate the percentage mediated. Abbreviations: CI, confidence interval; HOMA-IR, homeostatic model assessment insulin resistance; TBF, total body fat; VAT, visceral adipose tissue; HTGC, hepatic triglyceride content. Indirect effects were adjusted for sex, age, BMI at age 20, ethnicity, education, smoking, alcohol consumption, physical activity and family history of diabetes.

\section{Discussion}

The aim of our study was to investigate the association between adult weight change and insulin resistance at middle age, and to what extent this association is mediated by visceral fat and liver fat. In this population-based study of 1758 men and women, we observed that a gain in body weight during adulthood as small as $5 \%$ was already associated with more insulin resistance compared with weight maintenance during adulthood. Stronger associations with insulin resistance were observed for more excessive weight gain during adulthood. When considering the combined mediation effect, by adjusting for total body fat, visceral fat and liver fat in one model, we observed that the association between adult weight change and insulin resistance was $8.1 \%$ mediated by total body fat, $32.0 \%$ by visceral fat and $22.5 \%$ by liver fat. After adjustment for total body fat, visceral fat or liver fat separately, we observed the largest attenuation of the association between adult weight change and insulin resistance after adjustment for total body fat or visceral fat, compared with adjustment for liver fat. However, this results can be explained by the strong correlation between total body fat, visceral fat and liver fat, and thereby their overlapping mediation roles.

After adjustment for total body fat, visceral fat and liver fat at middle age, insulin resistance at middle age of individuals who had gained more than $50 \%$ of body weight was still 1.38 -fold higher than the insulin resistance of weight maintainers. This difference can be explained by either residual confounding or measurement error in the assessment of total body fat, because total body fat was estimated by bioelectrical impedance analysis (BIA). The estimation of total body fat percentage by BIA showed good absolute agreement (intraclass correlation coefficient $0.90,95 \%$ CI $0.89 ; 0.91$ ) with total body fat percentage by dual-energy X-ray absorptiometry (DXA) which was available in a small subset of the NEO study $(N=915)$. Alternatively, the remainder of the association between weight change and insulin resistance might be mediated by ectopic fat deposition in organs other than the liver, such as the heart, pancreas, kidneys, and skeletal muscles [18]. Because this information is not available in the NEO study, we were unable to investigate this hypothesis.

A meta-analysis based on 15 observational studies showed that the risk of type 2 diabetes mellitus was increasing in line with an increasing gain in body weight during adulthood, suggesting 
a dose-response association [35], in agreement with the results of our study. Also in line with our results, in a cross-sectional study of 153 middle-aged women HOMA-IR was higher in women who had gained more than $30 \mathrm{~kg}$ of body weight since the age of 20 than in women who had gained less than $10 \mathrm{~kg}$ [9]. A study in Japanese adults $(N=399)$ also showed that weight gain since age 20 years was associated with higher HOMA-IR [36]. The authors noted that this association attenuated after adjustment for BMI at middle age, suggesting that the association between weight gain and HOMA-IR was largely explained by the participants' BMI at middle age. In our study, we adjusted for total body fat, visceral fat and liver fat and showed that the association between adult weight change and insulin resistance is mostly mediated by visceral fat and liver fat.

The biological mechanism underlying these observations could be a reflection of the eventual limited capacity of subcutaneous adipose tissue to store lipids during weight gain $[14,18]$. When the capacity threshold of adipose tissue is reached, lipids will be stored in the visceral area and subsequently will be deposited in ectopic sites such as the liver, heart, muscles and pancreas [18,37]. Here, the visceral fat cells will exert their detrimental effects by secreting cytokines and non-esterified fatty acids (NEFAs) or very low-density lipoproteins [14,38-41]. Elevation in release of pro-inflammatory cytokines such as IL- 6 and TNF- $\alpha$ may induce a low-grade inflammatory state and oxidative stress, eventually leading to insulin resistance. Intracellular NEFAs inhibit insulin signaling, which will also lead to insulin resistance [42].

Strengths of our study include the large study population, data on many potential confounding factors, and the availability of directly assessed visceral adipose tissue by MRI and hepatic triglyceride content by ${ }^{1} \mathrm{H}-\mathrm{MRS}$, providing more accurate measures of abdominal adiposity than waist circumference. This enabled us to assess the mediating effects of visceral fat and liver fat after taking mediation via total body fat into account.

A limitation that needs to be considered is assessment of insulin resistance by HOMA-IR and Matsuda ISI. The golden standard measurement of insulin resistance is the hyperinsulinemic euglycemic clamp [43]. However, this is not feasible in a large study population. Instead, we calculated HOMA-IR and Matsuda ISI based on both fasting and postprandial glucose and insulin concentrations, which are valid surrogate measures for insulin resistance in large population-based studies [44]. The fact that the results of these two different proxies of insulin resistance were similar, suggests that our findings are robust. Second, we calculated BMI at age 20 using recalled weight at age 20 . Therefore, body weight at age 20 and weight change during adulthood might have been misclassified. However, previous studies have shown that recalled weight is highly correlated with measured weight at the same age [45], and in a previous study we showed that the association between adult weight change and measures of (abdominal) adiposity did not markedly change after correction for measurement error in recalled weight at age 20 years [16]. Third, the majority of our study population was Caucasian, therefore the results of our study need to be confirmed in other ethnic groups.

In conclusion, our results indicate that the association between adult weight gain and insulin resistance at middle age is largely mediated by visceral fat and liver fat at middle age, which is increasingly important given the growing prevalence of abdominal obesity and non-alcoholic fatty liver disease [46,47]. Our results suggest that weight maintenance during adulthood plays an important role in preventing accumulation of excess visceral fat and liver fat and thereby insulin resistance and eventually, type 2 diabetes at middle age and older age. Future prospective studies need to investigate the precise mechanisms by which visceral fat and liver fat lead to insulin resistance, and ways to reduce or prevent visceral fat and liver fat accumulation.

Supplementary Materials: The following are available online at http://www.mdpi.com/2077-0383/8/10/1559/s1, Table S1: Relative change with 95\% confidence intervals in measures of insulin resistance and sensitivity for categories of absolute weight change (in kilograms) during adulthood, compared with weight maintenance $(N=1758)$, Table S2: Association between of adult weight change (per 10\% change) and measures of insulin resistance and sensitivity, for complete study population and for men and women separately, Table S3: P-values for interaction between total body fat, visceral adipose tissue and hepatic triglyceride content and adult weight change in the association with measures of insulin resistance $(N=1758)$, Table S4: Mediation of the association between 
adult weight change and insulin resistance by total body fat, visceral adipose tissue and hepatic triglyceride content $(N=1758)$, Table S5: Mediation of the association between adult weight change and insulin resistance by total body fat, visceral adipose tissue and hepatic triglyceride content in men $(N=913)$, Table S6: Mediation of the association between adult weight change and insulin resistance by total body fat, visceral adipose tissue and hepatic triglyceride content in women $(N=845)$

Author Contributions: conceptualization, R.N., F.R.R., D.v.H., and R.d.M.; methodology, I.V., R.N., R.d.M., R.M.v.D., and S.l.C.; validation, I.V., R.N., and R.d.M.; formal analysis, I.V.; investigation, I.V., R.N., D.v.H., and R.d.M.; resources, H.J.L., R.d.M., and F.R.R.; writing—original draft preparation, I.V.; writing-review and editing, I.V., R.N., S.l.C., R.M.v.D., H.J.L., F.R.R., D.v.H., and R.d.M.; visualization, I.V.; supervision, R.N., F.R.R., D.v.H., and R.d.M.; project administration, I.V., R.N., and R.d.M.; funding acquisition, F.R.R.

Funding: The NEO study is supported by the participating Departments, the Division and the Board of Directors of the Leiden University Medical Centre, and by the Leiden University, Research Profile Area 'Vascular and Regenerative Medicine'. We acknowledge support from the Netherlands Cardiovascular Research Initiative: an initiative with support of the Dutch Heart Foundation (CVON2014-02 ENERGISE). We thank Nutricia Research, Utrecht, The Netherlands, for providing the mixed meal. D.V.H. was supported by the European Commission funded project HUMAN (Health-2013-INNOVATION-1-602757).

Acknowledgments: We express our gratitude to all individuals who participate in the NEO study. We are grateful to all participating general practitioners for inviting eligible participants. We furthermore thank P.R. van Beelen and all research nurses for collecting the data and P.J. Noordijk and her team for sample handling and storage and I. de Jonge, MSc for data management of the NEO study.

Conflicts of Interest: All authors declare that there is no conflict of interest associated with this manuscript.

\section{References}

1. Tirosh, A.; Shai, I.; Afek, A.; Dubnov-Raz, G.; Ayalon, N.; Gordon, B.; Derazne, E.; Tzur, D.; Shamis, A.; Vinker, S.; et al. Adolescent BMI trajectory and risk of diabetes versus coronary disease. N. Engl. J. Med. 2011, 364, 1315-1325. [CrossRef] [PubMed]

2. Abdullah, A.; Peeters, A.; De Courten, M.; Stoelwinder, J. The magnitude of association between overweight and obesity and the risk of diabetes: A meta-analysis of prospective cohort studies. Diabetes Res. Clin. Pract. 2010, 89, 309-319. [CrossRef] [PubMed]

3. Dale, C.E.; Fatemifar, G.; Palmer, T.M.; White, J.; Prieto-Merino, D.; Zabaneh, D.; Engmann, J.E.L.; Shah, T.; Wong, A.; Warren, H.R.; et al. Causal Associations of Adiposity and Body Fat Distribution With Coronary Heart Disease, Stroke Subtypes, and Type 2 Diabetes Mellitus: A Mendelian Randomization Analysis. Circulation 2017, 135, 2373-2388. [CrossRef] [PubMed]

4. De Mutsert, R.; Sun, Q.; Willett, W.C.; Hu, F.B.; van Dam, R.M. Overweight in early adulthood, adult weight change, and risk of type 2 diabetes, cardiovascular diseases, and certain cancers in men: A cohort study. Am. J. Epidemiol. 2014, 179, 1353-1365. [CrossRef] [PubMed]

5. Chan, J.M.; Rimm, E.B.; Colditz, G.A.; Stampfer, M.J.; Willett, W.C. Obesity, fat distribution, and weight gain as risk factors for clinical diabetes in men. Diabetes Care 1994, 17, 961-969. [CrossRef] [PubMed]

6. Colditz, G.A.; Willett, W.C.; Rotnitzky, A.; Manson, J.E. Weight gain as a risk factor for clinical diabetes mellitus in women. Ann. Intern. Med. 1995, 122, 481-486. [CrossRef] [PubMed]

7. Sun, W.; Shi, L.; Ye, Z.; Mu, Y.; Liu, C.; Zhao, J.; Chen, L.; Li, Q.; Yang, T.; Yan, L.; et al. Association between the change in body mass index from early adulthood to midlife and subsequent type 2 diabetes mellitus. Obesity 2016, 24, 703-709. [CrossRef] [PubMed]

8. Koh-Banerjee, P.; Wang, Y.; Hu, F.B.; Spiegelman, D.; Willett, W.C.; Rimm, E.B. Changes in Body Weight and Body Fat Distribution as Risk Factors for Clinical Diabetes in US Men. Am. J. Epidemiology 2004, 159, 1150-1159. [CrossRef] [PubMed]

9. Stefanska, A.; Sypniewska, G.; Blaszkiewicz, B.; Ponikowska, I.; Szternel, L.; Chojnowski, J. Long-term weight gain and metabolic syndrome, adiponectin and C-reactive protein in women aged 50-60 years. Adv. Med. Sci. 2010, 55, 186-190. [CrossRef]

10. Kissebah, A.H.; Peiris, A.N. Biology of regional body fat distribution: Relationship to non-insulin-dependent diabetes mellitus. Diabetes/Metab. Rev. 1989, 5, 83-109. [CrossRef]

11. Hartz, A.J.; Rupley, D.C.; Rimm, A.A. The association of girth measurements with disease in 32,856 women. Am. J. Epidemiol. 1984, 119, 71-80. [CrossRef] [PubMed] 
12. Landin, K.; Stigendal, L.; Eriksson, E.; Krotkiewski, M.; Risberg, B.; Tengborn, L.; Smith, U.; Landin-Wilhelmsen, K. Abdominal obesity is associated with an impaired fibrinolytic activity and elevated plasminogen activator inhibitor-1. Metabolism 1990, 39, 1044-1048. [CrossRef]

13. Kaye, S.A.; Folsom, A.R.; Sprafka, J.; Prineas, R.J.; Wallace, R.B. Increased incidence of diabetes mellitus in relation to abdominal adiposity in older women. J. Clin. Epidemiol. 1991, 44, 329-334. [CrossRef]

14. Després, J.-P.; Lemieux, I. Abdominal obesity and metabolic syndrome. Nature 2006, 444, 881-887. [CrossRef] [PubMed]

15. De Mutsert, R.; Gast, K.; Widya, R.; de Koning, E.; Jazet, I.; Lamb, H.; le Cessie, S.; de Roos, A.; Smit, J.; Rosendaal, F.; et al. Associations of Abdominal Subcutaneous and Visceral Fat with Insulin Resistance and Secretion Differ Between Men and Women: The Netherlands Epidemiology of Obesity Study. Metab. Syndr. Relat. Disord. 2018, 16, 54-63. [CrossRef] [PubMed]

16. Verkouter, I.; Noordam, R.; de Roos, A.; Lamb, H.J.; Rosendaal, F.R.; van Heemst, D.; de Mutsert, R. Adult weight change in relation to visceral fat and liver fat at middle age: The Netherlands epidemiology of obesity study. Int. J. Obes. 2019, 43, 790-799. [CrossRef] [PubMed]

17. Unger, R.H. Lipid overload and overflow: Metabolic trauma and the metabolic syndrome. Trends Endocrinol. Metab. 2003, 14, 398-403. [CrossRef] [PubMed]

18. Tchernof, A.; Despres, J.P. Pathophysiology of human visceral obesity: An update. Physiol. Rev. 2013, 93, 359-404. [CrossRef]

19. Klöting, N.; Fasshauer, M.; Dietrich, A.; Kovacs, P.; Schön, M.R.; Kern, M.; Stumvoll, M.; Blüher, M. Insulin-sensitive obesity. Am. J. Physiol. Endocrinol. Metab. 2010, 299, E506-E515. [CrossRef]

20. Targher, G.; Day, C.P.; Bonora, E. Risk of cardiovascular disease in patients with nonalcoholic fatty liver disease. N. Engl. J. Med. 2010, 363, 1341-1350. [CrossRef]

21. De Mutsert, R.; Den Heijer, M.; Rabelink, T.J.; Smit, J.W.A.; Romijn, J.A.; Jukema, J.W.; de Roos, A.; Cobbaert, C.M.; Kloppenburg, M.; le Cessie, S.; et al. The Netherlands Epidemiology of Obesity (NEO) study: Study design and data collection. Eur. J. Epidemiol. 2013, 28, 513-523. [CrossRef] [PubMed]

22. Matthews, D.R.; Hosker, J.P.; Rudenski, A.S.; Naylor, B.A.; Treacher, D.F.; Turner, R.C. Homeostasis model assessment: Insulin resistance and beta-cell function from fasting plasma glucose and insulin concentrations in man. Diabetologia 1985, 28, 412-419. [CrossRef] [PubMed]

23. Wallace, T.M.; Levy, J.C.; Matthews, D.R. Use and abuse of HOMA modeling. Diabetes Care 2004, 27, 1487-1495. [CrossRef] [PubMed]

24. Retnakaran, R.; Shen, S.; Hanley, A.J.; Vuksan, V.; Hamilton, J.K.; Zinman, B. Hyperbolic relationship between insulin secretion and sensitivity on oral glucose tolerance test. Obesity 2008, 16, 1901-1907. [CrossRef] [PubMed]

25. Matsuda, M.; DeFronzo, R.A. Insulin sensitivity indices obtained from oral glucose tolerance testing: Comparison with the euglycemic insulin clamp. Diabetes Care 1999, 22, 1462-1470. [CrossRef] [PubMed]

26. DeFronzo, R.A.; Matsuda, M. Reduced time points to calculate the composite index. Diabetes Care 2010, 33, e93. [CrossRef] [PubMed]

27. Verkleij-Hagoort, A.C.; de Vries, J.H.; Stegers, M.P.; Lindemans, J.; Ursem, N.T.; Steegers-Theunissen, R.P. Validation of the assessment of folate and vitamin B12 intake in women of reproductive age: The method of triads. Eur. J. Clin. Nutr. 2007, 61, 610-615. [CrossRef] [PubMed]

28. Wendel-Vos, G.W.; Schuit, A.J.; Saris, W.H.; Kromhout, D. Reproducibility and relative validity of the short questionnaire to assess health-enhancing physical activity. J. Clin. Epidemiol. 2003, 56, 1163-1169. [CrossRef]

29. De Hollander, E.L.; Zwart, L.; de Vries, S.I.; Wendel-Vos, W. The SQUASH was a more valid tool than the OBiN for categorizing adults according to the Dutch physical activity and the combined guideline. J. Clin. Epidemiol. 2012, 65, 73-81. [CrossRef]

30. Korn, E.L.; Graubard, B.I. Epidemiologic studies utilizing surveys: Accounting for the sampling design. Am. J. Public Health 1991, 81, 1166-1173. [CrossRef]

31. Lumley, T. Analysis of complex survey samples. J. Statist. Softw. 2004, 9, 1-19. [CrossRef]

32. Ministerie van Volksgezondheid, Welzijn en Sport. Nederland de Maat Genomen. Available online: www.rivm.nl/Onderwerpen/N/Nederland_de_Maat_Genomen (accessed on 13 August 2019).

33. Baron, R.M.; Kenny, D.A. The moderator-mediator variable distinction in social psychological research: Conceptual, strategic, and statistical considerations. J. Pers. Soc. Psychol. 1986, 51, 1173-1182. [CrossRef] [PubMed] 
34. Gunzler, D.; Chen, T.; Wu, P.; Zhang, H. Introduction to mediation analysis with structural equation modeling. Shanghai Arch. Psychiatry 2013, 25, 390-394. [PubMed]

35. Kodama, S.; Horikawa, C.; Fujihara, K.; Yoshizawa, S.; Yachi, Y.; Tanaka, S.; Ohara, N.; Matsunaga, S.; Yamada, T.; Hanyu, O.; et al. Quantitative relationship between body weight gain in adulthood and incident type 2 diabetes: A meta-analysis. Obes. Rev. 2014, 15, 202-214. [CrossRef] [PubMed]

36. Kimura, Y.; Pham, N.M.; Yasuda, K.; Nanri, A.; Kurotani, K.; Kuwahara, K.; Akter, S.; Sato, M.; Hayabuchi, H.; Mizoue, T. Association of adulthood weight gain with circulating adipokine and insulin resistance in the Japanese population. Eur. J. Clin. Nutr. 2015, 69, 462-466. [CrossRef] [PubMed]

37. Spalding, K.L.; Arner, E.; Westermark, P.O.; Bernard, S.; Buchholz, B.A.; Bergmann, O.; Blomqvist, L.; Hoffstedt, J.; Näslund, E.; Britton, T.; et al. Dynamics of fat cell turnover in humans. Nature 2008, 453, 783. [CrossRef] [PubMed]

38. Gustafson, B. Adipose tissue, inflammation and atherosclerosis. J. Atheroscler. Thromb. 2010, 17, $332-341$. [CrossRef] [PubMed]

39. Bergman, R.N.; Kim, S.P.; Catalano, K.J.; Hsu, I.R.; Chiu, J.D.; Kabir, M.; Hucking, K.; Ader, M. Why visceral fat is bad: Mechanisms of the metabolic syndrome. Obesity 2006, 14, 16S-19S. [CrossRef] [PubMed]

40. Jensen, M.D. Is visceral fat involved in the pathogenesis of the metabolic syndrome? Human model. Obesity. 2006, 14, 20S-24S. [CrossRef]

41. Kahn, S.E.; Hull, R.L.; Utzschneider, K.M. Mechanisms linking obesity to insulin resistance and type 2 diabetes. Nature 2006, 444, 840-846. [CrossRef]

42. Frayn, K. Adipose tissue as a buffer for daily lipid flux. Diabetologia 2002, 45, 1201-1210. [CrossRef] [PubMed]

43. DeFronzo, R.A.; Tobin, J.D.; Andres, R. Glucose clamp technique: A method for quantifying insulin secretion and resistance. Am. J. Physiol. 1979, 237, E214-E223. [CrossRef] [PubMed]

44. Bonora, E.; Targher, G.; Alberiche, M.; Bonadonna, R.; Saggiani, F.; Zenere, M.B.; Monauni, T.; Muggeo, M. Homeostasis model assessment closely mirrors the glucose clamp technique in the assessment of insulin sensitivity: Studies in subjects with various degrees of glucose tolerance and insulin sensitivity. Diabetes Care 2000, 23, 57-63. [CrossRef] [PubMed]

45. Casey, V.A.; Dwyer, J.T.; Berkey, C.S.; Coleman, K.A.; Gardner, J.; Valadian, I. Long-term memory of body weight and past weight satisfaction: A longitudinal follow-up study. Am. J. Clin. Nutr. 1991, 53, 1493-1498. [CrossRef] [PubMed]

46. WHO. Obesity and Overweight. Available online: www.who.int/mediacentre/factsheets/fs311/en/ (accessed on 9 September 2019).

47. Younossi, Z.M.; Koenig, A.B.; Abdelatif, D.; Fazel, Y.; Henry, L.; Wymer, M. Global epidemiology of nonalcoholic fatty liver disease-Meta-analytic assessment of prevalence, incidence, and outcomes. Hepatology 2016, 64, 73-84. [CrossRef] [PubMed]

(C) 2019 by the authors. Licensee MDPI, Basel, Switzerland. This article is an open access article distributed under the terms and conditions of the Creative Commons Attribution (CC BY) license (http://creativecommons.org/licenses/by/4.0/). 